\title{
Borrelia lusitaniae Infection Mimicking Headache, Neurologic Deficits, and Cerebrospinal Fluid Lymphocytosis
}

\author{
José Pedro Vieira, $M D^{1} \oplus$, Maria João Brito, $M D^{2}$, \\ and Isabel Lopes de Carvalho, PhD $^{3}$
}

\begin{abstract}
Headache with neurologic deficits and cerebrospinal fluid lymphocytosis (HaNDL) is a rare headache syndrome included in the Classification of Headache of the International Headache Society as a "headache attributed to non-infectious inflammatory intracranial disease."

We report one 15-year-old patient with clinical history and cerebrospinal fluid findings compatible with the diagnosis of HaNDL in whom Borrelia lusitaniae was identified in cerebrospinal fluid by polymerase chain reaction.
\end{abstract}

\author{
Keywords \\ encephalitis, meningitis, autoimmune, headache
}

Received February 3, 2019. Received revised April 20, 2019. Accepted for publication May 29, 2019.

Headache with neurologic deficits and cerebrospinal fluid lymphocytosis (HaNDL) is a headache syndrome included in the Classification of Headache of the International Headache Society as a "headache attributed to non-infectious inflammatory intracranial disease." 1 This uncommon syndrome is thought to result from transient immune-mediated central nervous system inflammation. ${ }^{1}$ Infectious agents were systematically looked for in patients' cerebrospinal fluid, using several techniques, almost always with negative results. ${ }^{1}$

The pathophysiology is incompletely understood. SPECT studies in symptomatic patients revealed very often localized hypoperfusion consistent with the focal neurologic findings presented by the patients. ${ }^{2}$ Reversible, focal, or even generalized slowing in EEGs are also consistent with limited regional cortical dysfunction. ${ }^{2}$

We report one 15-year-old patient with clinical history and cerebrospinal fluid findings compatible with the diagnosis of HaNDL in whom Borrelia lusitaniae was identified in cerebrospinal fluid by polymerase chain reaction (PCR).

\section{Case Report}

Our patient is a 15-year-old right-handed boy with a previous history of episodic headache with clinical characteristics of migraine without aura (infrequent, episodic, unilateral throbbing headache associated with nausea, photo and phonophobia). He was referred to our emergency department with a severe periorbital pain and frontal, left-sided, headache that had been present for several hours. He also had hypoesthesia and paresthesias in his right upper limb and later difficulty in speaking, and this was interpreted in the referring hospital as a confusional state.

He had no other relevant medical problems, and family history was negative for migraine. He lives in one region of Portugal where several Borrelia genospecies are endemic.

The patient was alert, oriented in time and place. Comprehension of spoken language was preserved but he had clear word-finding problems and a paraphasic speech consistent with motor aphasia. Repetition was preserved.

Meningeal signs were absent; cranial nerve examination was normal except for a questionable tongue deviation to the left. Tone, muscle strength, reflexes, and plantar responses were normal. Sensation was normal. The patient manifested a slight gait imbalance without any preponderant direction; dysmetria and intentional tremor were not present. No cutaneous lesions were apparent and there were no clinical signs of arthritis. Blood pressure was 140 to $80 \mathrm{~mm} \mathrm{Hg}$.

\footnotetext{
I Neurology Department, Hospital de Dona Estefânia, Lisbon, Portugal

${ }^{2}$ Infectious Diseases Unit, Hospital de Dona Estefânia, Lisbon, Portugal

${ }^{3}$ Infectious Diseases Department, National Institute of Health, Lisbon, Portugal

Corresponding Author:

José Pedro Vieira, MD, Neurology Department, Hospital de Dona Estefânia

Rua Jacinta Marto, I I69-045 Lisbon, Portugal.

Email: jose.vieira@chlc.min-saude.pt
} 
Computed tomography (CT) scan and later magnetic resonance imaging (MRI) with contrast were normal.

Cerebrospinal fluid analysis revealed 194 cells/ $\mu \mathrm{L}$ (lymphocytes), $53 \mathrm{mg} / \mathrm{dL}$ protein, and $53 \mathrm{mg} / \mathrm{dL}$ glucose. Blood glucose was $101 \mathrm{mg} / \mathrm{dL}$.

Cerebrospinal fluid culture was sterile. PCR for enterovirus, varicella-zoster, Epstein-Barr virus, cytomegalovirus, herpes simplex virus 1 and 2, human herpes virus 6 (HHV6), and human herpes virus 7 (HHV7) were negative. PCR was positive in cerebrospinal fluid for Borrelia burgdorferi sensu lato followed by a sequencing reaction that identified the genospecies B lusitaniae.

Serology for bartonella, brucella, and Epstein-Barr virus was negative. Enzyme-linked immunosorbent assay and immunoblot for Borrelia were negative. Venereal Disease Research Laboratory test was negative. Lupus anticoagulant was also negative.

Hemogram and renal and liver function tests were normal. C-reactive protein was $0.03 \mathrm{mg} / \mathrm{dL}$.

The patient was treated initially with intravenous ceftriaxone and acyclovir for presumed infectious encephalitis; completed a 21-day course of ceftriaxone; and recovered fully from headache, aphasia, and sensory symptoms within less than 24 hours. He remains asymptomatic with normal neurologic examination.

\section{Discussion}

Our patient had transiently a severe throbbing unilateral headache associated with sensory symptoms and aphasia, and cerebrospinal fluid examination disclosed a lymphocytic pleocytosis. This fulfilled, at the time of presentation, diagnostic criteria for HaNDL. ${ }^{1}$ He had a personal history of migraine, but in his present headache he did not report a visual aura and focal neurologic symptoms were reportedly concomitant with headache, both atypical features for migraine.

HaNDL was first described by Bartleson in $1981 .{ }^{3}$ Similar cases were subsequently reported defining more precisely the clinical picture. ${ }^{4}$ HaNDL is however a diagnosis of exclusion. Several diagnostic alternatives need to be considered in individual patients like meningoencephalitis (mainly herpes encephalitis), stroke, central nervous system vasculitis, and Mollaret meningitis.

In HaNDL, a unilateral throbbing headache is moderate to severe. Focal neurologic signs occur for up to hours. Hypo- or paresthesias usually in upper limbs are the most frequent neurologic symptoms, followed by aphasia and hemiplegia. Hemianopia is seen less often. Recurrence of headache is possible for as long as 3 months.

Etiology and pathophysiology of HaNDL are incompletely understood. Focal cortical dysfunction (focal slowing in EEG and focal hypoperfusion in SPECT) was demonstrated in almost all patients who have been investigated so far. ${ }^{2}$ Cerebrospinal fluid lymphocytic pleocytosis is frequently associated with elevated protein. Cerebrospinal fluid glucose is normal. Search for viral and bacterial agents has been almost uniformly negative. To our knowledge, in only a few patients one infectious agent was identified in association with HaNDL. ${ }^{5}$

$B$ lusitaniae is a genospecies of the Borrelia burgdorferi sensu lato complex and is the most prevalent Borrelia genospecies present in ticks in Portugal. The principal vector in Europe is Ixodes ricinus. ${ }^{6}$ This genospecies is prevalent in Mediterranean and North African countries and lizards appear to be the main reservoir. ${ }^{6}$ Pathogenicity for humans was at first considered unlikely. However, 2 case reports definitely implicate $B$ lusitaniae in human disease (skin lesion, cutaneous vasculitis and arthritis). ${ }^{6,7}$

The detection of antibodies against Borrelia by ELISA and even with immunoblot can be negative in patients who present neurologic impairment. PCR in cerebrospinal fluid samples has a low sensitivity, but it may be useful in very early Lyme neuroborreliosis with negative antibodies or in patients with immunodeficiency.

Moreover, our experience demonstrates that patients with $B$ lusitaniae infection will probably present as seronegative or with minimally positive antibody titers and their clinical symptoms and signs may be different from typical Lyme borreliosis and likely from neuroborreliosis.

The PCR from our patients cerebrospinal fluid was performed with a negative and a positive control, was repeated twice for confirmation and followed by sequencing. We did not repeat serologic tests for Borrelia once confirmation of the infection was established by molecular analysis.

Our patient's neurologic signs and symptoms were concomitant with a cerebrospinal fluid lymphocytic pleocytosis and molecular detection of $B$ lusitaniae. The disease course has probably been changed by antibiotic treatment.

Although a spontaneous cure and a benign course would not be expected with Neuroborreliosis, we hypothesize that other agents producing a self-limited infection may actually mimic HaNDL.

The most relevant aspect for the interpretation of our case would be, in our view, that some infections could transiently trigger focal cortical dysfunction and produce a clinical picture compatible with the diagnosis of HaNDL.

\section{Author Contributions}

All authors contributed to conception and design. JPV and MJB drafted the manuscrpt. All authors revised the final version and agree to be accountable for its content.

\section{Declaration of Conflicting Interests}

The authors declared no potential conflicts of interest with respect to the research, authorship, and/or publication of this article.

\section{Funding}

The authors received no financial support for the research, authorship, and/or publication of this article.

\section{ORCID iD}

José Pedro Vieira (D) https://orcid.org/0000-0002-2270-5314 
Ethical Approval/Institutional Review Board Approval

This paper was approved by our Institutional Ethics Committee (Document number 614/2018).

\section{References}

1. The International Classification of Headache Disorders, 3rd edition. Headache Classification Committee of the International Headache Society (IHS). Syndrome of transient headache and neurological deficits with cerebrospinal fluid lymphocytosis (HaNDL). Cephalalgia. 2018;38:10.

2. Fumal A, Vandenheede M, Coppola G, et al. The syndrome of transient headache with neurological deficits and CSF lymphocytosis (HaNDL): electrophysiological findings suggesting a migrainous pathophysiology. Cephalalgia. 2005;25:754-758.
3. Bartleson JD, Swanson JW, Whisnant JP. A migrainous syndrome with cerebrospinal fluid pleocytosis. Neurology. 1981;31:1257-1262.

4. Gomez-Aranda F, Canadillas F, Marti-Masso JF, et al. Pseudomigraine with temporary neurological symptoms and lymphocytic pleocytosis: a report of fifty cases. Brain. 1997;120:1105-1113.

5. Emond H, Schnorf H, Poloni C, et al. Syndrome of transient headache and neurological deficits with CSF lymphocytosis (HaNDL) associated with recent human herpesvirus-6 infection. Cephalalgia. 2009;29:487-491.

6. de Carvalho IL, Fonseca JE, Marques JG, et al. Vasculitis-like syndrome associated with Borrelia lusitaniae infection. Clin Rheumatol. 2008;27:1587-1591.

7. da Franca I, Santos L, Mesquita T, et al. Lyme borreliosis in Portugal caused by Borrelia lusitaniae? Clinical report on the first patient with a positive skin isolate. Wien Klin Wochenschr. 2005;117:429-432. 